\title{
Tipologia das rupturas de fala e classes gramaticais em crianças gagas e fluentes***
}

\author{
Typology of speech disruptions and grammatical classes in stuttering \\ and fluent children
}

Fabiola Juste*
Claudia Regina Furquim de Andrade**

*Fonoaudióloga. Doutora em Semiótica e Lingüística Geral pela Faculdade de Filosofia, Letras e Ciências Humanas da Universidade de São Paulo. Assistente do Laboratório de Investigação Fonoaudiológica da Fluência,

Motricidade e Funções Orofaciais do Departamento de Fisioterapia, Fonoaudiologia e Terapia Ocupacional da Faculdade de Medicina da Universidade de São Paulo. Endereço para correspondência: Av Paes de Barros, 2744 - Apto. 162 - São Paulo SP - CEP 03114-001 (fjuste@usp.br).

**Fonoaudióloga. Professora Titular do Departamento de Fisioterapia, Fonoaudiologia e Terapia Ocupacional da Faculdade de Medicina da Universidade de São Paulo.

****Parte da Tese de Doutorado da Primeira Autora Realizada no Laboratório de Investigação Fonoaudiológica da Fluência, Motricidade e Funções Orofaciais do Departamento de Fisioterapia, Fonoaudiologia e Terapia Ocupacional da Faculdade de Medicina da Universidade de São Paulo.

Artigo de Pesquisa

Artigo Submetido a Avaliação por Pares

Conflito de Interesse: não

\section{Abstract}

Background: developmental stuttering is a pathology which begins during childhood, during the phase of language acquisition and development and is characterized as being chronic. Aim: to verify the influence of typology and grammatical classes on the occurrence of speech disruptions of stuttering and fluent children. Method: participants of this study were 80 children, with ages between 4.0 and 11.11 years, residents in the city of São Paulo. Participants were divided in two groups: GI (research group) was composed by 40 children (29 male and 11 female) with the diagnosis of stuttering, and with no other associated communication, neurologic and cognitive deficits; GII (control group) was composed by 40 fluent children, paired by age and gender with the participants of GI. Results: the data indicate that the groups do not differ regarding the occurrence of typical disfluencies. Less typical disfluencies occurred predominantly for GI. As for the grammatical class, speech disruptions were more frequent in function words for both groups. Conclusion: these results indicate that the analyses of speech disruptions, in terms of typology and grammatical classes, bring several information that are necessary for the assessment and diagnosis of childhood stuttering. This analysis points the differences and similarities between stuttering and fluent children.

Key Words: Speech, Language and Hearing Sciences; Fluency; Stuttering; Speech; Childhood.

\section{Resumo}

Tema: a gagueira de desenvolvimento é aquela cujo surgimento se dá na infância, durante a fase de aquisição e desenvolvimento da linguagem, e que se caracteriza como uma desordem crônica. Objetivo: verificar a influência da tipologia e classe gramatical na ocorrência de rupturas na fala de crianças gagas e fluentes. Método: Participaram desse estudo 80 crianças, com idades entre 4.0 a 11.11 anos, residentes no município de São Paulo e Grande São Paulo. Os participantes foram divididos em dois grupos: GI (grupo de pesquisa) foi composto por 40 crianças, (29 do sexo masculino e 11 do sexo feminino) com diagnóstico de gagueira, sem qualquer outro déficit comunicativo, neurológico e cognitivo associado; GII (grupo controle) foi composto por 40 crianças fluentes, pareadas por idade e sexo aos participantes de GI. Resultados: os dados indicaram que os grupos não se diferenciaram quanto à ocorrência de rupturas comuns. As rupturas gagas ocorreram predominantemente para GI. Em relação à classe gramatical, as rupturas foram mais freqüentes nas palavras funcionais, para ambos os grupos. Conclusão: Esses resultados mostram que a análise das rupturas da fala, tanto em termos de tipologia quanto em termos gramaticais trazem um grande número de informações necessárias para a avaliação e diagnóstico da gagueira infantil, uma vez que aponta diferenças e semelhanças entre crianças gagas e fluentes.

Palavras-Chave: Fonoaudiologia; Fluência; Gagueira; Fala; Infância. 


\section{Introdução}

De acordo com Andrade (2003), as rupturas são os traços mais óbvios da gagueira e têm sido usadas como um parâmetro para descrever, definir e medir a gravidade dessa desordem. Como as rupturas também são encontradas na fala dos indivíduos fluentes, existe dificuldade em distinguir as rupturas normais daquelas que possam ser indicativas de gagueira.

Para Andrade (2003; 2004), as rupturas no fluxo da fala podem ser diferenciadas pela tipologia, ou seja, certas rupturas são comuns a todos os falantes e refletem fundamentalmente as incertezas e imprecisões lingüísticas, ou ainda, visam ampliar a compreensão da mensagem. Essas rupturas podem ser consideradas como comuns (hesitações, interjeições, revisões, palavras incompletas, repetições de palavras, segmentos e frases). Segundo a autora, existem algumas rupturas que, embora possam ocorrer esporadicamente para todos os falantes, são sugestivas de um maior comprometimento do processamento de fala. Essas rupturas são classificadas como rupturas gagas (repetições de sons e sílabas, prolongamentos, bloqueios, intrusões de sons e segmentos e pausas longas).

Assim como Andrade, Pereira (2003) também ressalta que existem vários tipos de rupturas que costumam estar presentes na fala das pessoas, sendo que o tipo e a freqüência de seu aparecimento são os fatores que irão diferenciar um falante fluente de um falante gago e para estes, indicar a gravidade do distúrbio.

Desde o início do século XX, estudos sugerem que a ocorrência de rupturas no discurso está relacionada a vários aspectos da formulação da linguagem.

Au-Yeung et al. (2003) sugerem que existe uma influência das classes gramaticais das palavras em relação à ocorrência de rupturas na fala. Em seu estudo, adotaram uma definição em relação às classes gramaticais, dividindo-as em palavras de conteúdo e funcionais.

As palavras de conteúdo (ou palavras de classe aberta) apresentam significado lexical e são palavras às quais, em princípio, sempre podem ser acrescentadas novas criações (Rosa, 2003). Essas palavras são os substantivos, os adjetivos, os verbos, os advérbios e os numerais. Essas palavras têm um papel fundamental na transmissão da informação semântica.

De acordo com Rosa (2003), os estudos tradicionais dedicam muita atenção às palavras de conteúdo. Elas são em maior número nas línguas (inclusive no Português), carregam significados, geram novos vocábulos. Os estudos de sintaxe têm, no entanto, demonstrado a importância de um outro grupo de palavras, as funcionais.

As palavras funcionais (ou palavras de classe fechada) apresentam um significado gramatical, elas são índices de propriedades gramaticais que fazem a diferença entre as línguas. Essas palavras são os artigos, as preposições, as conjunções, os pronomes e as interjeições. Essas palavras exercem principalmente funções sintáticas, servindo como elementos de ligação frasal com baixa carga semântica própria (Weber-Fox e Neville, 2001; Grela et al., 2004).

Em seu estudo, Au-Yeung et al. (2003) sugerem que as rupturas em palavras funcionais ocorrem principalmente quando essas precedem palavras de conteúdo na fala de crianças tanto fluentes quanto gagas. Os resultados comprovam a hipótese levantada no estudo de que as rupturas nas palavras funcionais são usadas como uma tática de adiamento quando a palavra de conteúdo subseqüente não está pronta para a execução.

Em um estudo posterior, Howell et al. (1999) pesquisaram a mudança na ocorrência de rupturas em palavras funcionais para palavras de conteúdo em falantes gagos e fluentes com o passar da idade. Os resultados indicaram que tanto crianças fluentes quanto gagas apresentaram maior número de rupturas em palavras funcionais. Com o aumento da idade, observa-se uma diferenciação entre os grupos. Os falantes fluentes continuam utilizando a tática do adiamento na produção da palavra de conteúdo, apresentando rupturas em palavras funcionais com o passar da idade. $\mathrm{O}$ grupo gago parece abandonar essa tática de adiamento e a partir da adolescência, começam a apresentar maior número de rupturas em palavras de conteúdo.

Em estudos publicados em 2002 e 2003, Dayalu et al. pesquisaram a ocorrência de rupturas na fala de adultos quanto à classe de palavras e os resultados apontam que o número de rupturas em palavras de conteúdo foi significativamente maior do que em palavras funcionais, sendo o mesmo resultado referido por Pereira (2003) em falantes adolescentes e adultos falantes do Português.

Segundo Bloodstein (2001), crianças tendem a apresentar maior número de rupturas em palavras funcionais, especialmente artigos, pronomes e conjunções, pois essas palavras geralmente iniciam frases. 
Mansur e Radanovic (2004) apontam que as palavras funcionais são exemplos de expressões que podem estar contidas dentro de um "pacote automático" (inseridas na frase já em fase final de processamento motor), enquanto as palavras de conteúdo, estarão sujeitas a uma elaboração mais refinada (cognitiva) e com maiores possibilidades de sofrer modificações circunstanciais.

Owens (1996) destaca que as palavras funcionais são produzidas mais tardiamente do que as palavras de conteúdo, sendo que as palavras funcionais tendem a ser omitidas nos primeiros segmentos produzidos pela criança. Segundo o autor, o crescimento lexical é lento até os dezoito meses de vida e que, nessa fase, o léxico de uma criança é composto por aproximadamente cinqüenta palavras. Estas palavras referem-se predominantemente a nomes de objetos (substantivos). A partir desse momento, a criança inicia um processo mais acelerado de aquisição. A aquisição de substantivos diminui e ocorre o crescimento na aquisição de verbos e palavras funcionais (principalmente artigos e pronomes).

Araújo (2003) em seu estudo realizado com 60 crianças falantes do Português com idades entre 2.0 a 4.11 anos sugere que os verbos são os vocábulos de maior ocorrência, seguidos pelos substantivos e pronomes. As classes gramaticais como artigo, preposição e conjunção também foram utilizadas pelas crianças, mas em menor número, quando comparadas às classes dos verbos, substantivos e pronomes.

A partir da literatura apresentada, esse estudo tem como objetivos: a) classificar, quantificar e comparar as tipologias de rupturas apresentadas na fala de crianças gagas e fluentes; b) verificar se as diferentes classes gramaticais de palavras interferem na ocorrência de rupturas de fala em gagas e fluentes.

As hipóteses de pesquisa testadas para responder aos objetivos propostos foram:

1. Quanto à ocorrência de rupturas, os grupos se diferenciarão quanto à freqüência e tipologia das rupturas apresentadas, sendo que as crianças gagas apresentarão um número maior de rupturas quando comparado ao grupo de crianças fluentes. 2. Quanto à classe de palavras, ambos os grupos apresentarão predominância de rupturas em palavras funcionais em relação às palavras de conteúdo.
3. A distribuição em palavras de conteúdo e funcionais será equivalente para as rupturas comuns e para as rupturas gagas para ambos os grupos.

\section{Método}

Os processos de seleção e avaliação seguiram os processos éticos pertinentes: Parecer da Comissão de Ética (CAPPesq HCFMUSP n ${ }^{\circ}$ 045/ 04) e a assinatura do Termo de Consentimento Livre e Esclarecido, pelos responsáveis dos participantes.

\section{Participantes}

Participaram dessa pesquisa 80 crianças na faixa etária de 4.0 a 11.11 anos de idade, de ambos os sexos ( 58 sexo masculino e 22 do sexo feminino), matriculados na rede pública de ensino, residentes no município de São Paulo e Grande São Paulo, cujos pais concordaram, através de assinatura do Termo de Consentimento, com a realização dos procedimentos propostos para realização da pesquisa.

Os participantes desse estudo foram divididos em dois grupos:

O grupo de pesquisa (GI) foi composto por 40 crianças (29 do sexo masculino e 11 do sexo feminino) com diagnóstico de gagueira, sem qualquer outro déficit comunicativo, neurológico e cognitivo associado. O diagnóstico de gagueira foi estabelecido segundo os critérios de avaliação adotados no Laboratório de Investigação Fonoaudiológica da Fluência, Motricidade e Funções Orofaciais (LIF - FMFO).

Os critérios de inclusão dos participantes do GI foram: apresentar pontuação do perfil da fluência fora dos valores de referência para a idade (Andrade, 2004); receber 11 pontos ou mais (gravidade equivalente a pelo menos "leve") no Stuttering Severity Instrument - 3 (SSI-3 - Riley, 1994).

O grupo controle (GII) foi composto por 40 crianças, pareadas ao grupo de pesquisa em relação à idade e sexo, sem queixa de gagueira e sem qualquer déficit comunicativo, neurológico e cognitivo, segundo informações fornecidas pelos pais.

Os critérios de inclusão dos participantes do GII foram: apresentar pontuação do perfil da fluência de acordo com os valores de referência para a idade (Andrade, 2004); receber um total de 10 pontos (gravidade equivalente a "muito leve") ou menos no SSI-3. 
Material

Para registro das amostras de fala, foram utilizados filmadora Panasonic NVRJ-28, um gravador (modelo Casio TP-6) como elemento de segurança para a garantia da qualidade da transcrição da fala, fitas cassete (TDK A-60) e fitas de vídeo (JVC TC-30).

As amostras de fala foram coletadas e analisadas quanto à tipologia das rupturas segundo o Protocolo do Perfil da Fluência (Andrade, 2004) e pelo Stuttering Severity Instrument - SSI (Riley, 1994), para determinar a gravidade de gagueira para GI.

\section{Procedimento}

1. Coleta das amostras de fala: para a obtenção das amostras de fala, adotou-se a metodologia proposta por Andrade (2004). Foi apresentada uma figura aos participantes e dada a seguinte ordem: "por favor olhe essa figura e me fale tudo o que você quiser sobre ela". O discurso só foi interrompido por perguntas e/ou comentários, nos casos em que houve a necessidade de incentivar a produção do discurso para a obtenção de 200 sílabas fluentes (número de sílabas fluentes necessárias para a análise da amostra). Quanto ao tempo previsto para cada coleta, foi estabelecido o mínimo de 5 minutos.

2. Levantamento das amostras de fala: após a coleta das amostras de fala dos participantes, as mesmas foram transcritas e caracterizadas segundo as tipologias das rupturas e classe gramatical. Em razão da grande variabilidade do número de informações e resultados gerados nas análises citadas acima, foi considerada necessária uma análise de confiabilidade. Para a avaliação da fidedignidade das análises realizadas, foi utilizada a validação dos resultados por compatibilização interjuízes, obtendo-se um índice de concordância de $96 \%$ para o juiz 1 e $92 \%$ para o juiz 2 .

2. A. Caracterização da tipologia das rupturas: as rupturas foram classificadas e analisadas segundo o Protocolo do Perfil da Fluência (Andrade, 2004), divididas em: rupturas comuns - hesitações, interjeições, revisões, palavras não terminadas, repetições de palavras, repetições de segmentos e repetições de frases; rupturas gagas - repetições de sons, repetições de sílabas, prolongamentos, bloqueios, pausas e intrusões.
2. B. Caracterização da classe gramatical das rupturas: as amostras, na sua totalidade (incluindo as rupturas), foram classificadas quanto à sua classe gramatical (substantivo, artigo, adjetivo, numeral, pronome, verbo, advérbio, preposição, conjunção e interjeição), segundo a Gramática Normativa proposta por Lima (2002).

Após essa classificação, as rupturas foram divididas em palavras de conteúdo (substantivos, adjetivos, verbos, advérbios e numerais) e palavras funcionais (artigos, preposições, conjunções, pronomes e interjeições), segundo os critérios adotados por Howell et al. (1999) e Shapiro e Caramazza (2002).

Para caracterizar cada ruptura quanto à sua classe gramatical e garantir a fidedignidade dessa categorização, foi utilizado como referência o dicionário Houaiss (2003).

Para essa análise, não foram consideradas todas as rupturas apresentadas pelos grupos, pois alguns tipos de rupturas como hesitação, palavra incompleta, pausa e intrusão não são classificáveis quanto à classe gramatical.

\section{Análise estatística}

Para a análise estatística dos dados, foram utilizados testes paramétricos (teste t-pareado, teste t-independente, análise de variância (ANOVA) e não paramétricos (teste de Friedman, teste de Mann-Whitney) com nível de significância de 5\%.

\section{Resultados}

De acordo com as hipóteses de pesquisa testadas, as amostras de fala foram analisadas e os resultados obtidos são apresentados nas Tabelas seguintes.

A Tabela 1 mostra que, para GI, houve um equilíbrio na distribuição do número de rupturas comuns e gagas, não apresentando diferença estatisticamente significante. Em relação ao GII, observa-se que o número de rupturas comuns foi aproximadamente dez vezes maior do que o número de rupturas gagas, sendo essa diferença estatisticamente relevante.

Comparando os grupos, observa-se que GI e GII não se diferenciaram estatisticamente quanto ao número de rupturas comuns. Quanto às rupturas gagas, houve uma diferenciação estatisticamente significante entre os grupos, sendo que GI apresentou um número aproximadamente treze vezes maior de rupturas gagas quando comparado a GII. 
TABELA 1. Comparação intra e intergrupos quanto à ocorrência de rupturas comuns e gagas.

\begin{tabular}{|c|c|c|c|c|c|c|c|c|c|c|}
\hline & \multicolumn{4}{|c|}{ Comuns } & \multicolumn{4}{|c|}{ Gagas } & \multicolumn{2}{|c|}{ P-Valor } \\
\hline & Total & Média & DP & Mediana & Total & Média & DP & Mediana & Teste-T & Mann-Whitney \\
\hline GI & 627 & 15,67 & 9,63 & 12,00 & 648 & 16,20 & 13,06 & 13,50 & 0,75 & 0,74 \\
\hline GII & 520 & 13,00 & 6,13 & 13,00 & 49 & 1,22 & 1,18 & 1,00 & $<0,001 *$ & $<0,001^{*}$ \\
\hline P-valor & & & & & & & & & & \\
\hline Teste-T & \multicolumn{4}{|c|}{0,143} & \multicolumn{4}{|c|}{$<0,001 *$} & & \\
\hline Mann-Whitney & \multicolumn{4}{|c|}{0,380} & \multicolumn{4}{|c|}{$<0,001 *$} & & \\
\hline
\end{tabular}

Legenda: DP - desvio-padrão.

A Tabela 2 apresenta os resultados referentes à ocorrência de rupturas comuns dos participantes, distribuídas em suas tipologias. Para GI, a média de ocorrências entre as tipologias comuns foi estatisticamente diferente, sendo que hesitação e repetição de palavras foram as tipologias de maior ocorrência, não diferenciandose entre si, mas diferenciando-se das demais tipologias comuns.

Em relação a GII, a distribuição da média de ocorrências entre as tipologias comuns também foi diferente estatisticamente, sendo a hesitação a tipologia de maior ocorrência (diferenciando-se das demais tipologias comuns).
Ambos os grupos apresentaram menor média de ocorrência da tipologia palavra incompleta e não apresentaram a tipologia de repetição de frase.

Para realizar uma comparação entre os grupos, cada uma das tipologias foi analisada separadamente. Os resultados indicam que os grupos não se diferenciaram estatisticamente quanto ao número de rupturas do tipo interjeição, revisão, palavra incompleta e repetição de frase. A ocorrência da tipologia hesitação foi maior para GII, sendo essa diferença estatisticamente relevante em relação a GI. As tipologias repetição de palavra e repetição de segmento também diferenciaram estatisticamente os grupos, apresentando maior ocorrência para GI.

TABELA 2. Rupturas comuns - distribuição intra e intergrupos

\begin{tabular}{|c|c|c|c|c|c|c|c|c|c|c|c|c|c|c|}
\hline & \multicolumn{2}{|c|}{ Hesitação } & \multicolumn{2}{|c|}{ Interjeição } & \multicolumn{2}{|c|}{ Revisão } & \multicolumn{2}{|c|}{ Pal. Incompleta } & \multicolumn{2}{|c|}{ Rep. Palavra } & \multicolumn{2}{|c|}{ Rep. Segmento } & \multicolumn{2}{|c|}{ P-Valor } \\
\hline & M & DP & M & DP & M & DP & M & DP & M & DP & M & DP & Teste-T & $\begin{array}{c}\text { Mann } \\
\text { Whitney }\end{array}$ \\
\hline GI & 4,67 & 4,16 & 0,97 & 1,42 & 1,57 & 1,58 & 0,30 & 0,56 & 6,60 & 6,00 & 1,55 & 1,58 & $<0,001 *$ & $<0,001 *$ \\
\hline $\begin{array}{c}\text { GII } \\
\text { P-valor }\end{array}$ & 6,32 & 4,00 & 1,45 & 2,26 & 1,25 & 1,48 & 0,47 & 0,87 & 2,87 & 2,55 & 0,62 & 0,80 & $<0,001 *$ & $<0,001 *$ \\
\hline Teste-T & \multicolumn{2}{|c|}{$0,075 *$} & \multicolumn{2}{|c|}{0,265} & \multicolumn{2}{|c|}{0,346} & \multicolumn{2}{|c|}{0,292} & \multicolumn{2}{|c|}{$<0,001 *$} & \multicolumn{2}{|c|}{$0,002 *$} & & \\
\hline $\begin{array}{c}\text { Mann } \\
\text { Whitney }\end{array}$ & \multicolumn{2}{|c|}{$\mathbf{0 , 0 3 3} *$} & \multicolumn{2}{|c|}{0,530} & \multicolumn{2}{|c|}{0,309} & \multicolumn{2}{|c|}{0,440} & \multicolumn{2}{|c|}{$<0,001 *$} & \multicolumn{2}{|c|}{$0,007 *$} & & \\
\hline
\end{tabular}

Legenda: M - média; DP - desvio-padrão; pal. - palavra; rep. - repetição.

A Tabela 3 apresenta os resultados referentes à ocorrência das rupturas gagas dos participantes distribuídas em suas tipologias. Observa-se, que para GI, a média de ocorrências entre as tipologias gagas foi estatisticamente diferente, sendo que repetição de sílaba, bloqueio e prolongamento foram as tipologias de maior ocorrência, não diferenciando-se entre si, mas diferenciando-se das demais tipologias gagas. As tipologias gagas com menor média de ocorrência foram a pausa e a intrusão.

Quanto a GII, a distribuição da média de ocorrências entre as tipologias comuns também foi estatisticamente diferente, sendo o prolongamento e a pausa as tipologias de maior ocorrência, não diferenciando-se entre si, mas diferenciando-se das demais tipologias gagas. A tipologia de menor média de ocorrência foi a repetição de som. As tipologias bloqueio e intrusão não foram observadas nas amostras analisadas desse grupo de crianças.

Comparando os grupos e analisando cada tipologia separadamente, observa-se que a ocorrência da pausa não diferenciou estatisticamente os grupos. As tipologias repetição de som, repetição de sílaba e prolongamento apresentaram maior ocorrência para GI, sendo essa diferença estatisticamente relevante. As tipologias bloqueio e intrusão não foram comparadas numericamente pois nenhuma ocorrência foi verificada nas amostras de fala de GII. 
TABELA 3. Rupturas gagas - distribuição intra e intergrupos

\begin{tabular}{|c|c|c|c|c|c|c|c|c|c|c|c|c|c|c|}
\hline & \multicolumn{2}{|c|}{ Rep. Som } & \multicolumn{2}{|c|}{ Rep. Sílaba } & \multicolumn{2}{|c|}{ Bloqueio } & \multicolumn{2}{|c|}{ Prolongamento } & \multicolumn{2}{|c|}{ Pausa } & \multicolumn{2}{|c|}{ Intrusão } & \multicolumn{2}{|c|}{ P-Valor } \\
\hline & M & $\mathrm{DP}$ & M & DP & M & DP & M & $\mathrm{DP}$ & M & DP & $\mathrm{M}$ & DP & Teste-T & $\begin{array}{c}\text { Mann } \\
\text { Whitney }\end{array}$ \\
\hline GI & 1,22 & 1,68 & 4,15 & 4,80 & 4,52 & 5,58 & 4,60 & 6,19 & 0,85 & 1,96 & 0,85 & 3,61 & & \\
\hline $\begin{array}{c}\text { GII } \\
\text { P-valor }\end{array}$ & 0,02 & 0,15 & 0,27 & 0,55 & 0 & 0 & 0,42 & 0,78 & 0,50 & 0,81 & 0 & 0 & & \\
\hline $\begin{array}{c}\text { Teste-T } \\
\text { Mann } \\
\text { Whitney }\end{array}$ & & & & & $\begin{array}{r}\text { nã } \\
\text { pos } \\
\text { real } \\
\text { dos } \\
\text { estatí } \\
\text { ausê } \\
\text { valor }\end{array}$ & $\begin{array}{l}\text { foi } \\
\text { el a } \\
\text { ação } \\
\text { stes } \\
\text { icos - } \\
\text { ia de } \\
\text { para } \\
\text { I }\end{array}$ & & & & & $\begin{array}{r}\text { na } \\
\text { pos } \\
\text { realiz } \\
\text { t } \\
\text { estat } \\
\text { ausê } \\
\text { valo }\end{array}$ & $\begin{array}{l}\text { foi } \\
\text { vel a } \\
\text { ão dos } \\
\text { es } \\
\text { icos- } \\
\text { ia de } \\
\text { para } \\
\text { I }\end{array}$ & & \\
\hline
\end{tabular}

Legenda: M - média; DP - desvio-padrão; rep. - repetição.

A Tabela 4 mostra que tanto GI quanto GII apresentaram predominância de palavras de conteúdo em relação às palavras funcionais em suas amostras, sendo essa diferença estatisticamente significante.

A Tabela aponta ainda que, nas amostras de fala analisadas, o número de palavras de conteúdo de GI não diferenciou-se estatisticamente do número de palavras de conteúdo de GII. Em relação às palavras funcionais, o mesmo resultado foi encontrado, ou seja, o número de palavras funcionais contidas nas amostras de GI não diferenciou-se estatisticamente de GII.

TABELA 4. Distribuição do número total de palavras da amostra quanto ao tipo de palavras.

\begin{tabular}{|c|c|c|c|c|c|c|c|c|c|c|}
\hline & \multicolumn{4}{|c|}{ Conteúdo } & \multicolumn{4}{|c|}{ Funcionais } & \multicolumn{2}{|c|}{ P-Valor } \\
\hline & Total & Média & DP & Mediana & Total & Média & DP & Mediana & Teste-T & Mann-Whitney \\
\hline GI & 2831 & 70,78 & 5,60 & 71,00 & 1773 & 44,33 & 7,51 & 45,00 & $<0,001^{*}$ & $<0,001 *$ \\
\hline GII & 2826 & 70,65 & 7,67 & 71,50 & 1748 & 43,70 & 10,9 & 44,00 & $<0,001 *$ & $<0,001 *$ \\
\hline P-valor & & & & & & & & & & \\
\hline Teste-T & \multicolumn{4}{|c|}{0,934} & \multicolumn{4}{|c|}{0,766} & & \\
\hline Mann-Whitney & \multicolumn{4}{|c|}{0,931} & \multicolumn{4}{|c|}{0,512} & & \\
\hline
\end{tabular}

Legenda: DP - desvio-padrão

A Tabela 5 apresenta o número total, média, desvio padrão e mediana das rupturas de fala das amostras que foram passíveis de análise quanto à sua classe gramatical e divididas em palavras de conteúdo e funcionais, segundo os critérios descritos no método desse estudo.

Apesar das amostras de fala apresentarem um número maior de palavras de conteúdo para ambos os grupos (Tabela 4), a Tabela 5 aponta que as rupturas de fala ocorreram em maior número nas palavras funcionais, também para ambos os grupos. Tanto para GI quanto para GII, o predomínio de rupturas nas palavras funcionais apresentou diferença estatisticamente significante em relação às rupturas em palavras de conteúdo. 
TABELA 5. Distribuição do número de rupturas em palavras de conteúdo e funcionais.

\begin{tabular}{|c|c|c|c|c|c|c|c|c|c|c|}
\hline & \multicolumn{4}{|c|}{ Conteúdo } & \multicolumn{4}{|c|}{ Funcionais } & \multicolumn{2}{|c|}{ P-Valor } \\
\hline & Total & Média & DP & Mediana & Total & Média & $\mathrm{DP}$ & Mediana & Teste-T & Mann-Whitney \\
\hline GI & 491 & 12,23 & 9,56 & 9,00 & 630 & 15,8 & 11,9 & 12,50 & $\mathbf{0 , 0 0 7} *$ & 0,077* \\
\hline GII & 91 & 2,00 & 1,88 & 2,00 & 216 & 5,73 & 1,88 & 5,00 & $<0,001 *$ & $<0,001 *$ \\
\hline \multicolumn{11}{|l|}{ P-valor } \\
\hline Teste-T & \multicolumn{4}{|c|}{$<0,001 *$} & \multicolumn{4}{|c|}{$<0,001 *$} & & \\
\hline Mann-Whitney & \multicolumn{4}{|c|}{$<0,001 *$} & \multicolumn{4}{|c|}{$<0,001 *$} & & \\
\hline
\end{tabular}

Legenda: DP - desvio-padrão.

Analisando os grupos em relação à quantidade, GI apresentou maior número de rupturas, tanto em palavras de conteúdo quanto em palavras funcionais, quando comparado a GII.

A Tabela 6 apresenta os resultados quanto à ocorrência de rupturas em palavras de conteúdo, distribuídas em suas classes gramaticais. Descartando-se as diferenças numéricas entre os grupos, observa-se que tanto para GI quanto para GII, o verbo foi a classe gramatical que mais apresentou rupturas (diferenciando-se estatisticamente das demais classes gramaticais).

TABELA 6. Distribuição das rupturas em palavras de conteúdo de acordo com sua classe gramatical

\begin{tabular}{|c|c|c|c|c|c|c|c|c|c|c|c|c|}
\hline & \multicolumn{2}{|c|}{ Substantivo } & \multicolumn{2}{|c|}{ Adjetivo } & \multicolumn{2}{|c|}{ Verbo } & \multicolumn{2}{|c|}{ Advérbio } & \multicolumn{2}{|c|}{ Numeral } & \multicolumn{2}{|c|}{ P-Valor } \\
\hline & M & $\mathrm{DP}$ & M & $\mathrm{DP}$ & M & $\mathrm{DP}$ & M & $\mathrm{DP}$ & $\mathrm{M}$ & $\mathrm{DP}$ & ANOVA & Friedman \\
\hline GI & 3,57 & 4,67 & 0,17 & 0,38 & 6,37 & 4,41 & 2,05 & 2,82 & 0,05 & 0,22 & $<0,001 *$ & $<0,001 *$ \\
\hline GII & 0,62 & 0,83 & 0 & 0 & 1,05 & 1,46 & 0,25 & 0,66 & 0,07 & 0,26 & $<0,001 *$ & $<0,001 *$ \\
\hline
\end{tabular}

Legenda: M - média; DP - desvio-padrão.

A Tabela 7 apresenta os resultados quanto à ocorrência de rupturas em palavras funcionais, distribuídas em suas classes gramaticais. Novamente descartando -se as diferenças numéricas entre os grupos, os resultados indicaram que o artigo foi a classe gramatical que mais apresentou rupturas para ambos os grupos (diferenciando-se estatisticamente das demais classes gramaticais).

TABELA 7. Distribuição das rupturas em palavras funcionais de acordo com sua classe gramatical

\begin{tabular}{cc|c|c|c|c|c|c|c|c|c|c|c}
\hline & \multicolumn{2}{c|}{ Pronome } & \multicolumn{2}{c|}{ Preposição } & \multicolumn{2}{c|}{ Conjunção } & \multicolumn{2}{c|}{ Artigo } & \multicolumn{2}{|c|}{ Interjeição } & \multicolumn{2}{c}{ P-Valor } \\
\hline & M & DP & M & DP & M & DP & M & DP & M & DP & ANOVA & Friedman \\
\hline GI & 3,30 & 2,84 & 2,92 & 3,62 & 2,95 & 3,90 & 5,62 & 5,71 & 0,97 & 1,42 & $<\mathbf{0 , 0 0 1 *}<\mathbf{0 0 0 0 1}^{*}$ \\
GII & 0,75 & 1,19 & 0,67 & 0,85 & 0,97 & 1,67 & 1,90 & 1,49 & 1,42 & 2,27 & $<\mathbf{0 , 0 0 1 *}<$ & $<\mathbf{0 , 0 0 1 *}$ \\
\hline
\end{tabular}

Legenda: M - média; DP - desvio-padrão. 
A Tabela 8 mostra que, para ambos os grupos, as rupturas comuns ocorreram em maior número nas palavras funcionais em relação às palavras de conteúdo, sendo essa diferença estatisticamente significante.
Na Tabela 9 observa-se que, para ambos os grupos, as rupturas gagas distribuíram-se de forma equivalente em palavras de conteúdo e palavras funcionais, ou seja, não houve um predomínio de rupturas estatisticamente significante em um desses grupos de palavras.

TABELA 8. Distribuição das rupturas comuns em palavras de conteúdo e funcionais.

\begin{tabular}{|c|c|c|c|c|c|c|c|c|c|c|}
\hline \multicolumn{11}{|c|}{ Rupturas comuns } \\
\hline & \multicolumn{4}{|c|}{ Conteúdo } & \multicolumn{4}{|c|}{ Funcionais } & \multicolumn{2}{|c|}{ P-Valor } \\
\hline & total & Média & DP & Mediana & Total & Média & DP & Mediana & Teste-T & Mann-Whitney \\
\hline GI & 146 & 3,65 & 2,82 & 3,00 & 371 & 9,28 & 7,34 & 7,50 & $<0,001 *$ & $<0,001 *$ \\
\hline GII & 77 & 1,93 & 1,80 & 2,00 & 201 & 6,30 & 4,03 & 6,00 & $<0,001 *$ & $<0,001 *$ \\
\hline
\end{tabular}

Legenda: DP - desvio-padrão.

TABELA 9. Distribuição das rupturas gagas em palavras de conteúdo e funcionais.

\begin{tabular}{|c|c|c|c|c|c|c|c|c|c|c|}
\hline \multicolumn{11}{|c|}{ Rupturas Gagas } \\
\hline & \multicolumn{4}{|c|}{ Conteúdo } & \multicolumn{4}{|c|}{ Funcionais } & \multicolumn{2}{|c|}{ P-Valor } \\
\hline & Total & Média & DP & Mediana & Total & Média & $\mathrm{DP}$ & Mediana & Teste-T & Mann-Whitney \\
\hline G I & 345 & 8,62 & 8,63 & 6,00 & 259 & 6,48 & 6,70 & 4,00 & 0,090 & 0,084 \\
\hline G II & 14 & 0,35 & 0,57 & 0,00 & 15 & 0,37 & 0,74 & 0,00 & 0,860 & 0,814 \\
\hline
\end{tabular}

Legenda: DP - desvio-padrão.

\section{Discussão}

Hipótese 1: confirmada.

Os resultados apresentados mostraram que os grupos se diferenciaram quanto ao número total de rupturas apresentadas, sendo que o grupo de crianças gagas apresentou mais do que o dobro de rupturas quando comparado ao grupo de crianças fluentes.

Analisando a distribuição das rupturas, observou-se que os grupos apresentam comportamentos diferenciados. Para as crianças gagas, houve um equilíbrio na distribuição do número de rupturas comuns e gagas. Em relação às crianças fluentes, houve um significativo predomínio do número de rupturas comuns quando comparado ao número de rupturas gagas.

Apesar das rupturas gagas serem um dos mais importantes parâmetros para o diagnóstico da gagueira (Yairi, 1999, 2001;Andrade, 2003 ), observase que elas também estão presentes na fala de crianças fluentes. O que diferencia os grupos é a freqüência com que essas rupturas ocorrem na fala dessas crianças (Yairi, 1999). Em uma pesquisa realizada com 200 crianças fluentes falantes do Português, Andrade (2003) aponta que em nenhuma das idades pesquisadas, o número de rupturas gagas nas amostras de fala foi superior a cinco ocorrências.

Em relação às rupturas comuns, os resultados indicaram que os grupos não se diferenciaram quanto ao número de ocorrência. A diferenciação entre os grupos ocorreu em relação ao número de rupturas gagas (aproximadamente 13 vezes maior para as crianças gagas). Esses achados estão de acordo com Ambrose e Yairi (1999) uma vez que, em relação às tipologias do tipo OD (other disfluencies), as diferenças entre os grupos não foi considerada estatisticamente relevante e as rupturas do tipo SLD (stuttering-like disfluencies) diferenciam os grupos, sendo maior para as crianças gagas. 
Apesar dos grupos não apresentarem diferenças estatisticamente significantes quanto ao número total de ocorrências de rupturas comuns, observa-se uma diferenciação quanto à distribuição dessas rupturas em suas tipologias.

Dentre as tipologias consideradas comuns, a repetição de palavras e a hesitação foram as de maior ocorrência para as crianças gagas. Para Anderson e Conture (2005), das tipologias não indicativas de gagueira, a repetição de palavra foi a tipologia de maior ocorrência em seu estudo realizado com 20 crianças gagas falantes do Inglês.

Para as crianças fluentes, a hesitação foi a tipologia comum de maior ocorrência. No estudo de Yairi (1999), as tipologias hesitação e interjeição foram as de maior ocorrência tanto para crianças gagas quanto fluentes. Para Carlo e Watson (2003), que realizaram um estudo com crianças fluentes falantes do Espanhol, as rupturas consideradas comuns mais frequentes foram a repetição de palavra, revisões e hesitações.

Fazendo uma análise em cada tipologia isoladamente, os resultados indicaram que o número de ocorrências das tipologias revisão, palavra incompleta e interjeição não apresentaram diferenças estatisticamente significantes para os grupos de crianças gagas e fluentes.

As tipologias de repetição de palavra e repetição de segmento diferenciaram estatisticamente os grupos, apresentando maior ocorrência para as crianças gagas. Outra tipologia comum que diferenciou estatisticamente os grupos foi a hesitação; porém, dessa vez, sua ocorrência foi maior para as crianças fluentes.

Na literatura internacional, alguns autores fazem uma clara distinção entre a repetição de palavras monossílabas e as demais, sendo a primeira, classificada como tipologia característica de gagueira.

Yairi (2001), respondendo a uma crítica feita por Wingate, defende a posição de que a repetição de palavras monossílabas deve ser considerada como uma ruptura gaga. Segundo o autor, a fala não é separada em sílabas e palavras, pois existe a coarticulação entre elas. Dessa forma, não existe diferença entre a repetição da palavra monossílaba (ex. a a a boat) e a repetição de sílaba (ex. aaabout) pois a quebra na coarticulação ocorre da mesma forma. Em seus estudos (Yairi, 1999) o grupo de crianças gagas apresentou predomínio estatisticamente significante de repetição de palavras monossílabas comparado ao grupo de crianças fluentes.

Uma consideração que pode ser feita a essa teoria é que ela se refere à todas as palavras monossílabas, mas suas justificativas e exemplificações parecem referir-se sempre aos artigos, que não são as únicas palavras monossílabas, tanto da língua Inglesa quanto das demais línguas.

As teorias mais modernas sobre gagueira concordam que a ocorrência de rupturas na fala decorre de um desajuste temporal, uma assincronia entre os processamentos envolvidos na fala (Perkins et al.,1991; Andrade, 2002; Howell, 2004).

A teoria proposta por Postma e Kolk (1993) baseia-se na noção de que dificuldades na codificação fonológica e na habilidade de construir e recuperar o plano fonético, podem resultar em rupturas na fala. De acordo com essas teoria, pessoas que gaguejam parecem apresentar uma lentidão no processo de codificação fonológica, aumentando a chance de erro na seleção do fonema alvo. O reflexo da tentativa de reparação desses erros seriam as ruptura no fluxo da fala.

Segundo Levelt (1989), de um modo geral, o funcionamento da codificação fonológica até sua execução é decorrente do preenchimento das fendas, estruturas já existentes, pelos conteúdos que são processados a cada nível: fonético, fonológico, sintático e semântico.

De acordo com a teoria estruturalista, para Saussure (1969), as relações entre os elementos lingüísticos podem ser estabelecidas em dois domínios distintos, o eixo da seleção (paradigmático) e o eixo das combinações (sintagmático). O paradigma não é qualquer associação de signos pelo som ou pelo sentido, mas uma série de elementos lingüísticos suscetíveis de figurar no mesmo ponto do enunciado, se o sentido for outro. Por outro lado, no sintagma não se combinam quaisquer elementos aleatoriamente, a combinação no sintagma obedece a um padrão definido pelo sistema. Assim, por exemplo, podem-se combinar um artigo e um substantivo e, nesse caso, o artigo deve sempre preceder o substantivo.

A teoria estruturalista coloca a linearidade como uma das características essenciais do signo lingüístico, onde cada signo ocupa uma posição determinada no plano sintagmático, não podendo, por exemplo, haver a pronúncia de dois fonemas na mesma unidade de tempo. A comutação é a operação que põe em funcionamento ambos os eixos.

Dessa maneira, uma falha na temporalização dos processamentos envolvidos na fala pode refletir na seleção dos eixos paradigmáticos e sintagmáticos, dificultando a seleção do termo subseqüente, impedindo a linearidade do sistema, podendo causar repetições de termos, ou seja, rupturas na fala.

As repetições de palavras e segmentos apresentadas em maior número para as crianças gagas fragmentam um elemento maior da fala, o sintagma (nominal ou verbal), o que pode significar que o 
indivíduo apresenta dificuldade em iniciar e manter uma estrutura sintática inteira (Bloodstein, 2001).

As crianças fluentes deste estudo apresentaram um número significativamente maior de rupturas do tipo hesitação quando comparadas às crianças gagas. Pensando na relação sintagmática/paradigmática discutida acima, as hesitações comportam-se de maneira diferente se comparadas às repetições de palavras e segmentos. A hesitação não causa a repetição de elementos do sintagma e, dessa forma, não rompe o mesmo. A ocorrência das hesitações pode ser entendida como um tempo adicional necessário para o ajuste temporal dos eixos paradigmáticos e sintagmáticos.

Em relação às tipologias gagas, os resultados indicaram diferenças entre os grupos.

Para as crianças gagas, as tipologias de maior ocorrência foram repetição de sílaba, bloqueio e prolongamento, não havendo diferença estatisticamente relevante entre elas. O mesmo resultado foi encontrado por Anderson e Conture (2005). Para Yairi (1999), a ruptura de maior ocorrência foi a repetição de palavras monossílabas, considerada por esse autor como ruptura gaga, como já discutido anteriormente. As tipologias gagas de menor ocorrência foram a pausa e a intrusão.

Em relação às crianças fluentes, as tipologias gagas de maior ocorrência foram o prolongamento e pausa. No estudo de Carlo e Watson (2003) o prolongamento foi a ruptura mais freqüente. A tipologia de menor ocorrência foi a repetição de som. As tipologias bloqueio e intrusão não foram observadas nas amostras analisadas desse grupo de crianças.

Fazendo uma comparação entre os grupos e analisando cada tipologia separadamente, observase que as tipologias repetição de som, repetição de sílaba e prolongamento apresentaram maior ocorrência para as crianças gagas, sendo essa diferença estatisticamente relevante.

Para as tipologias bloqueio e intrusão não foi possível realizar a comparação já que as crianças fluentes não apresentaram essas rupturas.

A única tipologia gaga que não apresentou diferença estatisticamente relevante entre os grupos foi a pausa. Nesse caso, outros aspectos devem ser pesquisados, como o local e a duração da pausa, a fim de verificar possíveis diferenças entre a ocorrência dessa tipologia em crianças gagas e fluentes.

Hipótese 2: confirmada.

Os resultados obtidos neste estudo mostram que apesar das amostras de fala apresentarem um número maior de palavras de conteúdo, as rupturas de fala ocorreram em maior número nas palavras funcionais, para ambos os grupos. Esse resultado corrobora com os achados amplamente destacado na literatura internacional (Howell et al., 1999; Bloodstein, 2001; Au-Yeung et al., 2003; Dworzynski et al., 2003; Graham et al., 2004; Natke et al., 2004).

Segundo Au-Yeung et al., (2003) as rupturas em palavras funcionais acontecem quando essas precedem palavras de conteúdo na fala de crianças tanto gagas quanto fluentes, pois as rupturas nas palavras funcionais são usadas como uma tática de adiamento quando a palavra de conteúdo subseqüente não está pronta para a execução.

De acordo com Howell et al. (1999), somente as palavras de conteúdo são realmente palavras no sentido fonológico, sendo que as palavras funcionais servem de afixos às palavras de conteúdo. Por esse motivo, quando o falante apresenta rupturas sucessivas de palavras funcionais, ele está na verdade, recomeçando a sentença e atrasando a produção da palavra de conteúdo subseqüente, aumentando assim seu tempo de processamento.

Analisando a ocorrência de rupturas para cada classe gramatical individualmente, observou-se um comportamento muito parecido entre crianças gagas e fluentes, pois ambos os grupos apresentaram predominância de rupturas nas classes gramaticais verbo (palavras de conteúdo) e artigo (palavras funcionais).

Em relação à classe gramatical dos verbos, a grande ocorrência de rupturas pode ser explicada pelo fato do verbo ser uma classe gramatical bastante complexa e dinâmica. A complexidade dos verbos é observada tanto sintática como morfologicamente. Os verbos são adquiridos mais tardiamente, são mais difíceis de serem processados por apresentarem uma grande variação de significados. Além disso, apresentam maior dificuldade de serem compreendidos quando comparados aos substantivos. O processamento de verbos é mais complexo do que o processamento de substantivos em decorrência da grande variabilidade de flexões e da quantidade de informação (gramatical e temática) representada nos verbos (Bi et al 2005; Goldberg e Golfarb, 2005; Honincthun e Pillon, 2005).

Em relação aos artigos, além da alta freqüência dessa classe gramatical no Português (Rosa, 2003), o expressivo número de rupturas em artigos pode ter sido influenciado por dois motivos. O primeiro motivo seria que no Português, existe uma alta freqüência de artigos iniciando frases (Rosa, 2003). Para Bloodstein (2001), crianças tendem a apresentar maior número de rupturas em palavras funcionais, especialmente artigos, pronomes e conjunções, pois essas palavras 
geralmente iniciam frases. No início da sentença, o planejamento emissão ainda não está completado, sendo que os falantes podem modificar o planejamento inicial durante a fala, acarretando uma maior exigência do sistema lingüístico, facilitando a ocorrência de rupturas (Karniol, 1995). Segundo Pereira (2003), as palavras iniciais de uma frase são mais suscetíveis de serem gaguejadas do que as demais, demonstrando uma dificuldade maior em iniciar uma expressão verbal. Isso não significa que não possam ocorrer rupturas no meio ou no final da frase, mas estas são menos freqüentes.

O segundo motivo, como já citado anteriormente, seria que as rupturas em palavras funcionais, principalmente nos artigos, parecem ter a finalidade de atrasar a produção da palavra de conteúdo seguinte até que ela esteja pronta para a produção (Howell et al., 1999; Au-Yeung et al., 2003). A predominância de rupturas na classe gramatical de artigos é amplamente divulgada na literatura (Bloodstein, 2001; Au-Yeung et al., 2003; Dworzynski et al., 2003; Graham et al., 2004; Natke et al., 2004).

Hipótese 3: parcialmente confirmada.

Analisando o cruzamento entre a ocorrência de rupturas comuns e gagas, de acordo com a classe gramatical (palavras de conteúdo e funcionais), foi observado um dado interessante. De acordo com os resultados obtidos, observou-se que as rupturas comuns ocorreram em maior número (mais do que o dobro) em palavras funcionais. Para as rupturas gagas, a ocorrência em palavras de conteúdo e funcionais foi a mesma, não havendo predomínio para nenhum dos dois tipos de palavras.

De acordo com Andrade (2004), as rupturas comuns estão mais relacionadas com o processamento lingüístico. Segundo a autora, a ocorrência excessiva das rupturas comuns pode ser indicativo de que o sistema lingüístico excedeu sua capacidade em achar palavras ou formular sentenças, em gerar emissões socialmente apropriadas através da sequencialização de sílabas fonologicamente corretas e em gerar combinações fonológicas complexas (Andrade, 2002).

\section{Referências Bibliográficas}

AMBROSE, N. G.; YAIRI, E. Normative data for early childhood stuttering. J. Lang. Hear. Res., v. 42, p. 895-909, 1999.

ANDERSON, J. D.; CONTURE, E. G. Language abilities of children who stutter: a preliminary study. J. Fluenc. Dis., v. 25, p. 269-290, 2005.
Desse modo, os resultados sugerem que o aspecto gramatical, representado pela diferenciação entre palavras de conteúdo e funcionais, parece influenciar de alguma forma a ocorrência de rupturas comuns, já que foi observado um significativo predomínio desse tipo de ruptura em palavras funcionais.

As rupturas gagas, por sua vez, parecem não sofrer essa influência gramatical da mesma forma que as rupturas comuns, já que a distribuição dessas rupturas em palavras de conteúdo e funcionais foi a mesma, não apresentando diferenças estatisticamente significantes.

Esse resultado sugere que o aspecto gramatical representado pela diferenciação entre palavras de conteúdo e funcionais parece não influenciar diretamente na ocorrência das rupturas gagas, mais relacionadas aos aspectos motores envolvidos na fala.

Qualquer situação que demande do processamento motor habilidades que excedam sua capacidade fisiológica, acarretará num aumento do número desse tipo de ruptura (gaga). A demanda motora significa qualquer ocorrência que torne a movimentação suave e rápida das estruturas faciais, necessárias para a produção da fala fluente, mais difícil (Andrade, 2002).

É importante ressaltar que essa hipótese de pesquisa é uma proposta pioneira, já que estudos similares não foram encontrados na literatura pesquisada, impossibilitando a comparação dos achados.

\section{Conclusão}

Esses resultados mostram que a análise das rupturas da fala, tanto em termos de tipologia quanto em termos gramaticais trazem um grande número de informações necessárias para a avaliação e diagnóstico da gagueira infantil, uma vez que aponta diferenças e semelhanças entre crianças gagas e fluentes, separando aspectos que são pertinentes apenas à gagueira dos aspectos inerentes ao desenvolvimento da linguagem infantil.

ANDRADE, C. R. F. História natural da gagueira - estudo III: vocabulário, fonologia e pragmática. Pró-fono R. Atual. Cient., Barueri (SP), v. 14, n. 3, p. 371-382, 2002.

ANDRADE, C. R. F. Gagueira Infantil. In: ANDRADE, C. R. F; MARCONDES, E. (Orgs). Fonoaudiologia em Pediatria. São Paulo: Sarvier, 2003. p. 61-69. 
ANDRADE, C. R. F. Fluência. In: ANDRADE, C. R. F.; BÉFI-LOPES, D. M.; WERTZNER, H. F.; FERNANDES, F. D. M. $A B F W$ - teste de linguagem infantil: nas áreas de fonologia, vocabulário, fluência e pragmática. 2. ed. Barueri (SP): Pró-Fono, 2004. cap. 3, p. 71-94.

ARAÚJO, K. Aspectos do desempenho gramatical de crianças pré-escolares em desenvolvimento normal de linguagem. 2003. 223 f. Dissertação (Mestrado em Semiótica e Lingüística Geral) - Faculdade de Filosofia, Letras e Ciências Humanas, Universidade de São Paulo, São Paulo.

AU-YEUNG, J.; GOMEZ, I. V.; HOWELL, P. Exchange of disfluency with age from function words to content words in Spanish speakers who stutter. J. Speech Lang. Hear. Res., v. 46, p. 754-766, 2003.

BI, Y.; HAN, Z.; SHU, H.; CARAMAZZA, A. Are verbs like inanimate objects? Brain Lang., v. 95, p. 28-29, 2005.

BLOODSTEIN, O. Incipient and developed stuttering as two distinct disorders: resolving a dilemma. J. Fluenc. Dis., v. 26, p. $67-73,2001$.

CARLO, E. J.; WATSON, J. B. Disfluencies of 3 - and 5 years old Spanish-speaking children. J. Fluenc. Dis., v. 28, p. 37-53, 2003.

DAYALU, V. N.; KALINOWSKI, J.; STUART, A.; HOLBERT, D.; RASTATTER, M. P. Stuttering frequency on content and function words in adults who stutter: a concept revised. J. Speech Lang. Hear. Res., v. 45, p. 871-878, 2002.

DAYALU, V. N.; KALINOWSKI, J.; STUART, A. Interpreting differences in stuttering frequency on content and function words: a reply to Wingate (2003). J. Speech Lang. Hear. Res., v. 46, p. 1471-1472, 2003.

DWORZYNSKI, K.; HOWELL, P.; NATKE, U. Predicting stuttering from linguistics factors for German speakers in two age groups. J. Fluenc. Dis., v. 28, p. 95-113, 2003.

GOLDBERG, E.; GOLDFARB, R. Grammatical category ambiguity in aphasia. Brain Lang., v. 95, p. 293-303, 2005.

GRAHAM, C. G.; CONTURE, E. G.; CAMARATA, S. M. Chilhood stuttering on function and content words. In: ASHA CONVENTION, 2004. On-line proceedings of Asha Convention, 2004. Disponível em URL: <http:// convention.asha.org/handouts.cfm>. Acesso em: 29 nov. 2004.

GRELA, B.; RASHITI, L.; SOARES, M. Dative prepositions in children with specific language impairment. Applied Psycholing., v. 25, p. 467-480, 2004.

HONINCTHUN, P.; PILLON, A. Why verbs could be more demanding of executive resources than nouns: insight from a case study of a fv-FTD patient. Brain Lang., v. 95, p. 3637, 2005.

HOUAISS, A. Minidicionário Houaiss da Língua Portuguesa. Rio de Janeiro: Objetiva, 2003.
HOWELL, P. Assessment of some comtemporary theories of stuttering that applied to spontaneous speech. Contemp. Issues Commun. Sci. Dis., v. 39, p. 122-139, 2004.

HOWELL, P.; AU-YEUNG, J.; SACKIN, S. Exchange of stuttering from function words to content words with age. J. Speech Lang. Hear. Res., v. 42, p. 345-354, 1999.

KARNIOL, R. Stuttering, language and cognition: a review and model of stuttering as suprasegmental sentence plan alignment (SPA). Pychol. Bull, v. 117, n. 1, p. 104-124, 1995.

LEVELT, W. J. M. Speaking: from intention to articulation. Cambridge (MA): MIT Press, 1989.

LIMA, C. H. R. Gramática normativa da língua portuguesa. 42. ed. Rio de Janeiro: José Olympio, 2002.

MANSUR, L. L.; RADANOVIC, M. Neurolingüística: princípios para a prática clínica. São Paulo: EI - Edições Inteligentes, 2004.

NATKE, U.; SANDRIESER, P.; ARK, M.; PIETROWSKY, R.; KALVERAM, K. T. Linguistic stress, within-word position, and grammatical class in relation to early childhood stuttering. J. Fluenc. Dis., v. 29, p. 109-122, 2004.

OWENS, R. E. Language development. 4. ed. Needhan Height: Allyn \& Bacon, 1996.

PEREIRA, M. M. B. Análise lingüística da gagueira. São Paulo: AM3 Artes, 2003.

PERKINS, W. H.; KENT, R. D.; CURLEE, R. F. A theory of neuropsycholinguistic function in stuttering. J. Speech Hear. Res., v. 34, p. 734-752, 1991.

POSTMA, A.; KOLK, H. The covert repair hypothesis: prearticulatory repair processes in normal and stuttered disfluencies. J. Speech Hear. Res., v. 36, p. 472-487, 1993.

RILEY, G. D. A Stuttering Severity Instrument for children and adults. Austin: Pro-Ed, 1994.

ROSA, M. C. Classes de palavras, tipos de significado e questões relacionadas. In: ROSA, M. C. Introdução à Morfologia. São Paulo: Contexto, 2003. p. 91-114.

SAUSSURE, F. Curso de lingüística geral. São Paulo: Cultrix, 1969.

SHAPIRO, K.; CARAMAZZA, A. The role and neural representation of grammatical class. J. Neuroling., v. 15, p. 159-170, 2002. special issue.

WEBER-FOX, C.; NEVILLE, H. Sensitive periods differentiate processing of open - and closed - class words: an ERP study of bilinguals. J. Speech Lang. Hear. Res., v. 44, p. 1338-1353, 2001

YAIRI, E.; WATKINS, R.; AMBROSE, N.; PADEN, E. Letters to the Editor. J. Speech Lang. Hear. Res., v. 44, p. 585-597, 2001 